\title{
HIGH POWER COUPLER ISSUES IN NORMAL CONDUCTING AND SUPERCONDUCTING ACCELERATOR APPLICATIONS
}

\author{
H. Matsumoto, KEK, Tsukuba, 305, Japan
}

\begin{abstract}
The ceramic material $\left(\mathrm{Al}_{2} \mathrm{O}_{3}\right)$ commonly used for the klystron output coupler in normal conducting, and for an input coupler to superconducting cavities is one of the most troublesome parts in accelerator applications. But the performance can be improved very much by starting with high purity (>99.9\%) alumina powder of controlled grainsize $(0.1 \sim 0.5-\mu \mathrm{m})$, and reducing the magnesium $(\mathrm{Mg})$ sintering-binder to lower the dielectric loss to the order of $10^{-}$ ${ }^{4}$ at S-band frequencies. It has been confirmed that the new ceramic can stand a peak S-band frequency rf power of up to $300 \mathrm{MW}$ and $2.5 \mu \mathrm{sec}$ pulse width.
\end{abstract}

\section{INTRODUCTION}

This study was carried out at KEK by the author and Dr. A. Miura, with the collaboration of Mr. W. R. Fowkes and Mr. R. S. Callin of SLAC.

The motivation of this study was to clarify the origin of discharge breakdowns of the alumina ceramic material in rf windows, and to demonstrate a breakthrough that allows use of $\mathrm{rf}$ windows for peak rf power exceeding 100 MW. Originally the breakdown mechanisms were not fully understood; but since light emission from the alumina ceramic disk was observed, multipactoring phenomenon was suspected [1]. Therefore many studies were done to study local heat build-up due to multipactoring [2], [3]. Considerable progress was made through efforts at SLAC [4], and Varian Co., Inc. A variety of surface-coating materials were tested as possible cures. Coating with TiN, which has a low secondary electron emission coefficient and good chemical stability, proved effective [5], [6]. However, more recently it was discovered at SLAC that even a TiN coated disk can be destroyed by discharge breakdown, most often when the rf power transmission exceeds $50 \mathrm{MW}$. In this power regime no clear correlation was found between light emission and breakdown. The problem was regarded mostly as a technical issue for the coating, and again many studies were undertaken [7], [8], [9]. From a literature survey and from examination of destroyed windows, we have come to suspect that high power breakdown of alumina disks may be due to phenomena taking place inside the alumina rather than (or besides) at its surface. The root cause of the problem might be voids within the alumina or structural defects due to binder materials used during the sintering process. Thus, the origins of destruction of the ceramic disk have to be separated into two regimes: one for the low peak rf power with cw or millisecond long pulses, and the other for high peak rf power with pulses on the order of several tens of microseconds.

In this paper I will describe the destruction breakdown mechanism of alumina ceramic in the rf window as elucidated by simulation and experiment. Further, results of this study can be used to improve the ceramic material of input couplers for superconducting cavities.

\section{ELECTRON MULTIPACTORING}

We investigated the behavior of multipactor electrons on the rf window under various power transmission conditions. Then comparisons were made between the theoretical expectations and the actual experimental results on samples.

We used an rf window that would make it simple to study the electron multipactor and the discharge breakdown of ceramic disks. A de-mountable pill-box type rf window was developed to avoid the brazing that can cause discharge breakdown at the junction between the disk and the enclosure at high peak rf power. The window consists of an alumina disk in a pair of stainless steel enclosures that are copper plated $(\sim 20 \mu \mathrm{m})$ on their inner surfaces, as shown in Figure 1.

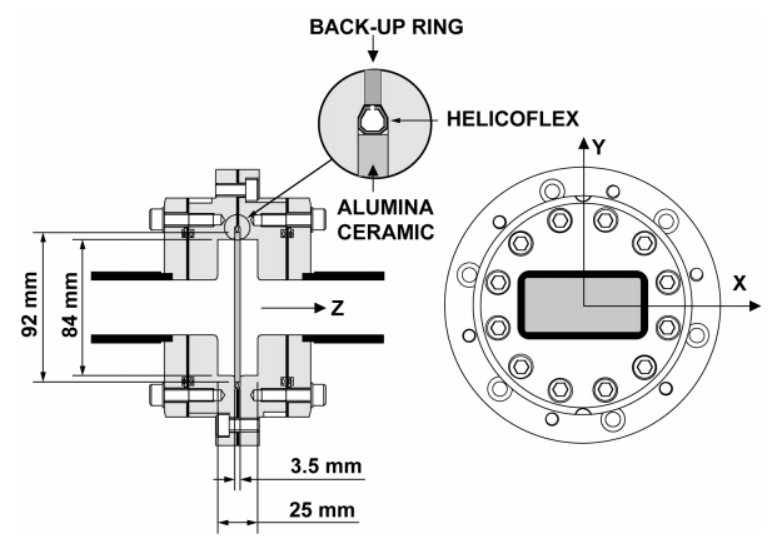

Figure 1: A cross-sectional view of the de-mountable type rf-window used in testing. The measured input VSWR is 1.05 or less at $2856 \mathrm{MHz} \pm 50 \mathrm{MHz}$.

Figure 2 shows the propagation modes in a typical pillbox type rf window as calculated by the MAFIA 3D-code. As can be seen in the figure, the propagation modes through the rf window are $\mathrm{TE}_{11}$-like and $\mathrm{TM}_{11}$-like. Therefore, there are two electric field patterns on the ceramic disk that are parallel and then perpendicular to the disk which appear alternately. The perpendicular electric field (Fig. 2-b) causes most discharge breakdowns of ceramic disks, such as pinhole punctures and cracks. We have extended the original simulation code [10] in a few areas to suit our purposes.

Figure 3 shows a single-surface multipactoring electron orbit calculated during the simulation [11]. It shows that the free electron path is at most a few $\mathrm{cm}$. Cathode luminescence of alumina is known to occur with incident electron energies below a few tens of $\mathrm{keV}$. Thus the experimentally observed light emission from alumina windows is consistent with cathode luminescence due to bombardment of 
multipactoring electrons.
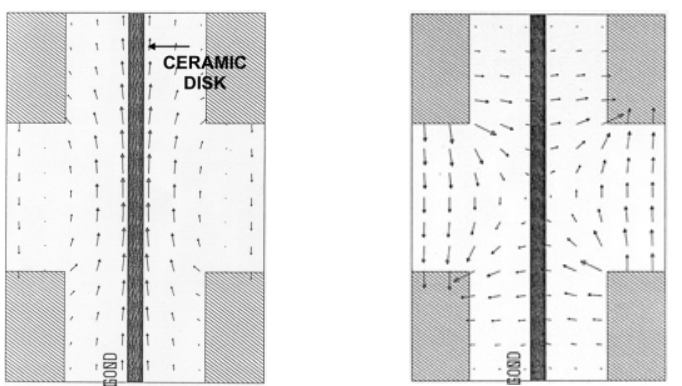

(a) Side view of $\mathrm{TE}_{11}$-like parallel (b) Side view of the $\mathrm{TM}_{11}$-like electric field on the ceramic disk. perpendicular electric field on the ceramic disk.

Figure 2: Propagating mode through the rf window. The arrows show the electric field direction.

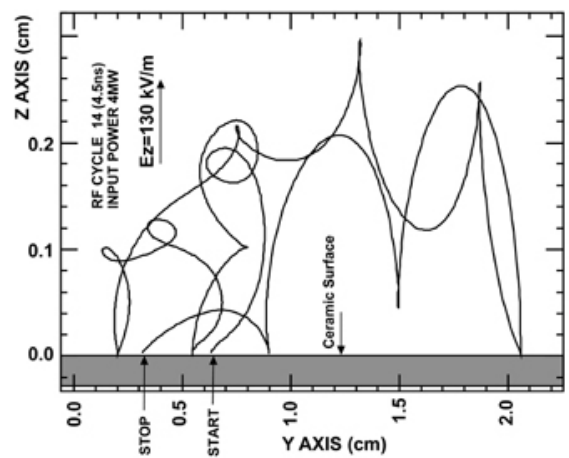

Figure 3: Typical single-surface multipactoring with $4 \mathrm{MW}$ rf power transmission through the window. ( $\mathrm{Y}$ and $\mathrm{Z}$ are defined in Fig. 1). The curve is a projection of 3-dimensional electron trajectory onto the $\mathrm{Y}-\mathrm{Z}$ plane. The free electron path in the $\mathrm{X}$ direction is $\varnothing 10 \mathrm{~mm}$.

Figures 4-a1, -a2 and -a3 show the density distribution of electrons hitting the ceramic surface. Figures 4-b1, -b2 and -b3 show the light emission pattern observed during a high power test. The calculated multipactor region and the light emission pattern are in qualitatively good agreement.

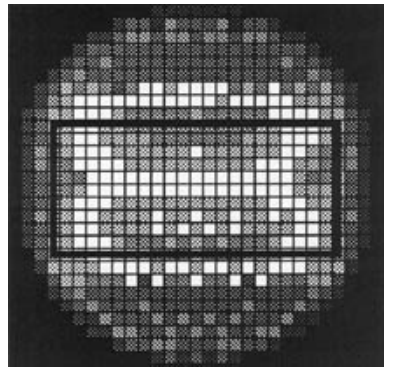

(a1)

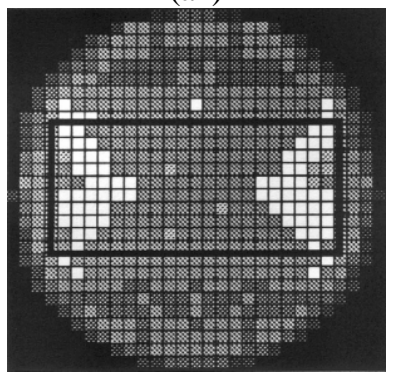

(a2)

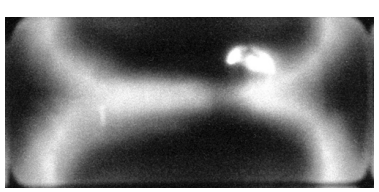

(b1)

Transmission rf power of $4 \mathrm{MW}$ for $2.5 \mu \mathrm{sec}$.

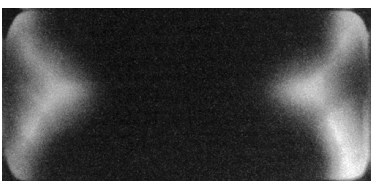

(b2)

Transmission rf power of $10 \mathrm{MW}$ for $2.5 \mu \mathrm{sec}$.

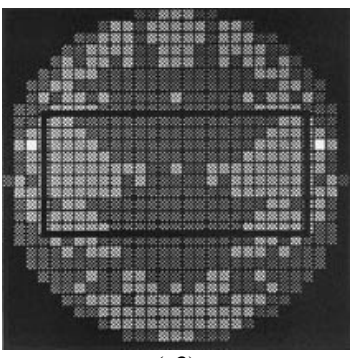

(a3)

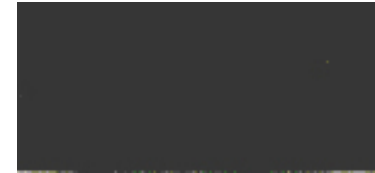

(b3)

Transmission rf power of $50 \mathrm{MW}$ for $2.5 \mu \mathrm{sec}$.

Figure 4: Left shows the calculated distributions of multipactoring on the alumina ceramic disk surface. The gray scale darkness indicates the electron intensity in 5 steps, $0-100 \%$. Right: shows the luminescence from the surface of the alumina ceramic disk in our experiment. None of the samples has any coating such as TiN.

According to the calculations, multipactoring is predicted to be basically non-existent at any transmission power above $50 \mathrm{MW}$, as shown in Figure 4-a3. Most likely this is because multipactoring depends on the $E \perp$, the strength of the electric field component perpendicular to the alumina surface. Our calculation shows that multipactoring is strongest when $E \perp$ is $100-450 \mathrm{kV} / \mathrm{m}$. When the transmission power is above 50 MW, $E \perp$ exceeds $450 \mathrm{kV} / \mathrm{m}$ and a resonant condition favorable for multipactoring no longer obtains, because the electrons accelerated along $E \perp$ can reach the opposite copper surface and be absorbed during a half rf cycle. As seen in Figures 5 and 12 (lower traces), the multipactoring areas disappear in a high perpendicular electrical field $(E \perp)$.

This leads us to conclude that when an alumina disk fails in a high power environment (transmission power $>50 \mathrm{MW}$ ), it would be unreasonable to take multipactoring as the primary cause. Some other factors should be investigated as the root causes of breakdown of alumina disks at high rf power. Voids and impurities in the alumina are possibilities. However, even at high peak rf power levels, it was found that multipactor occurs during the rf pulse transient time (Fig. 5). Therefore, coating with TiN is necessary to maintain the best possible overall characteristics of alumina ceramic for $\mathrm{rf}$ windows.

From this, we may conclude that the TiN coating is very effective for low peak power and long pulse applications, such as for the input coupler to a superconducting cavity.

Figure 5 shows the typical time profile of the light emission from an alumina ceramic disk.

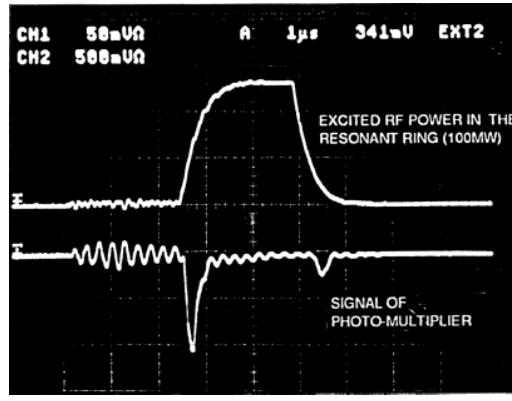

Figure 5: Upper trace: Time profile of rf power transmission through the rf window with alumina ceramic disk for sample 3. Lower trace: Signal from the photo-multiplier monitoring the light emission from the disk.

The light emission signal trace is synchronized with the transmission rf power profile. At the rising and falling edges 
of the rf pulse, when the power transmission is close to 10 MW, light emission for a few hundred nano-seconds is clearly seen. Note that our sample did not have a TiN coating.

We understand that although the accelerating operational power was well above the multipactor regime, during the pulse transient time the disk momentarily experiences the power level where multipactoring is prominent. Therefore, for $\mathrm{rf}$ window disks in actual use, a TiN coating is required to reduce the charge on the alumina ceramic disk due to electron multipactoring during the pulse transient time.

\section{ALUMINA CERAMIC MATERIAL IMPROVEMENT}

\subsection{High-Purity Fine Alumina Powder and HIP}

Typical disks for windows have been made of $99.5 \%$ purity alumina ceramics, in which the $0.5 \%$ of impurities act to reduce the sintering temperature to around $1400-1500{ }^{\circ} \mathrm{C}$. Sintering binders such as magnesium $(\mathrm{Mg})$ comprise the major part of the impurities that keep the alumina grain size to around 10 to $20 \mu \mathrm{m}$ after sintering. If the binder fraction is made smaller, the sintering temperature has to be increased up to $1600{ }^{\circ} \mathrm{C}$, and this increases the prosity (void population). Thus, reduction of impurities and reduction of porosity are incompatible. Consistent with this, we found experimentally that high purity $(99.9 \%)$ alumina disks do not perform much better in a high power environment [9]. Figure 6 shows photographs of typical alumina ceramic samples.

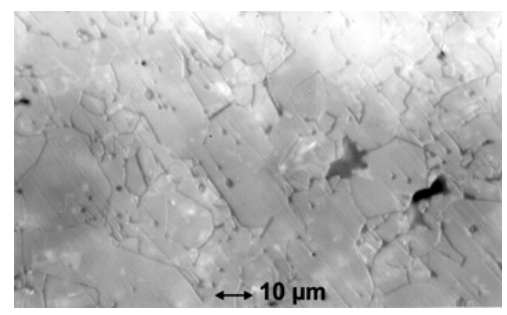

Figure 6: Photographs of alumina ceramics disks for $\mathrm{rf}$ windows. Voids within the alumina or structural defect are Black.

These problems have two possible solutions. The first is to use very fine alumina powder. If the powder size is smaller, the sintering temperature can be lowered while using only a small amount of binder. Recent progress in the alumina industry has resulted in high purity $(99.9 \%)$ ultra fine alumina powders of 0.1-0.5 $\mu \mathrm{m}$ diameter, as shown in Fig. 7. Low temperature sintering of such fine powder reduces the void population.

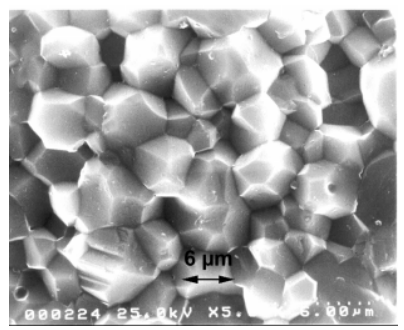

Figure 7: Electron microscope photographs of alumina ceramic, made from ultra fine $(\varnothing 0.1-0.5 \mathrm{~mm})$ and high purity $(99.9 \%)$ alumina powder, showing the uniform grain size and the absence of large voids at grain boundaries.

The second solution is to apply Hot Isostatic Pressing (HIP), a thermo-mechanical process that applies gas pressure at high temperatures to attain high density [12]. The voids in alumina materials are removed ("squeezed out") in a high temperature $\left(1400{ }^{\circ} \mathrm{C}\right)$, high pressure $\left(2000 \mathrm{kgf} / \mathrm{cm}^{2}\right)$ environment in an inert gas such as argon, as shown in Fig.8.

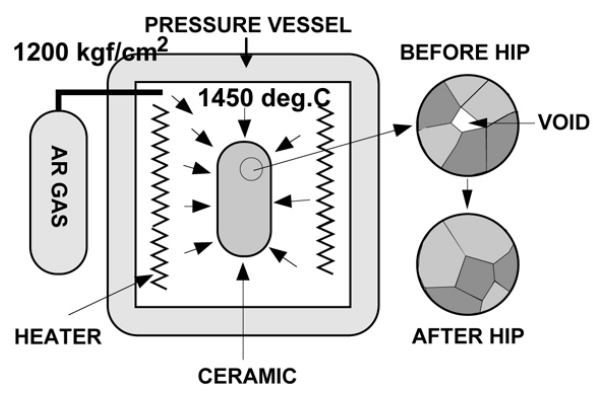

Figure 8: Schematic diagram of a HIP treatment set-up. The furnace is inside the pressure vessel, and it is set to keep the temperature well below the melting point of the material being processed.

This treatment can reduce the alumina ceramic porosity to a negligibly small $0.5 \%$. The alumina grains are tightly packed. This also improves the mechanical strength. The improvements due to HIP treatment are summarized in Table 1 and the results, are shown in Fig. 9.

Table 1: Properties of the alumina ceramic disk before and after the HIP treatment.

\begin{tabular}{|l|c|c|}
\hline & Before HIP & After HIP \\
\hline Porosity (\%) (volume/weight) & 3.8 & 0.5 \\
\hline Fracture strength (kgf/cm ${ }^{2}$ ) & 29.8 & 36.1 \\
\hline Dielectric constant (at $2853 \mathrm{MHz})$ & 9.4 & 9.7 \\
\hline
\end{tabular}

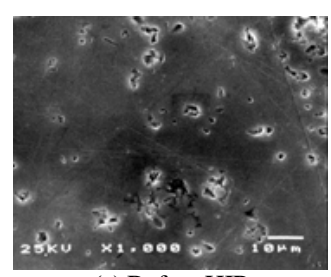

(a) Before HIP

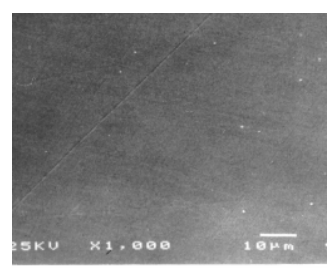

(b) After HIP
Figure 9: Electron microscope photographs of alumina ceramic before and after HIP treatment $\left(1450{ }^{\circ} \mathrm{C}, 2000 \mathrm{kgf} / \mathrm{cm}^{2}, 2\right.$ hours): (a) shows voids at the alumina grain boundaries; (b) shows almost no voids in any area.

The light transparency of the alumina is also increased. Impurities or voids become easily identifiable, because they appear as dark clouds. This allows for easy visual inspection for rejection of undesirable alumina ceramic disks before use, as shown in Fig. 10.

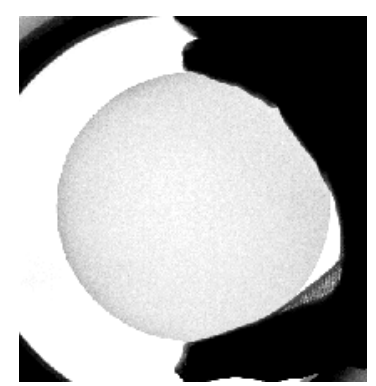

(a) Clear

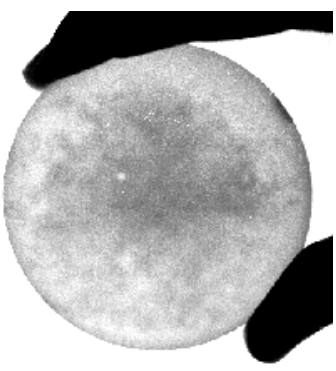

(b) Clouded
Figure 10: Comparison of clear and clouded alumina ceramic disks. The samples, of $3.5 \mathrm{~mm}$ thickness and $92 \mathrm{~mm}$ diameter, are held up to an incandescent light $(100 \mathrm{~W}, 10 \mathrm{~cm}$ behind $)$.

\subsection{Sintering Binder and RF Loss}


The rf loss of alumina ceramic disks is affected by the amount and composition of impurities. To function as an rf window the rf loss should be kept low of course. The rf loss due to binders has been found to depend strongly on the choice of binder materials [13]. For example, it is very sensitive to the presence of $\mathrm{MgO}$. We have fabricated alumina ceramic disks of varying purity with and without $\mathrm{MgO}$ and measured their characteristics. Table 2 shows the results. With no contain $\mathrm{MgO}$ at all, a very low dielectric loss is achieved. Table 2 shows the results.

Table 2: Physical properties of high-purity alumina ceramic.

\begin{tabular}{|l|c|c|c|}
\hline Alumina ceramic purity & $99.5 \%$ & $99.9 \%$ & $99.9 \%$ \\
\hline $\mathrm{MgO}$ content $(\%)$ & 0.19 & 0.06 & 0 \\
\hline $\begin{array}{l}\tan \delta\left(\times 10^{-4}\right): \\
(\text { at } 2853 \mathrm{MHz})\end{array}$ & 13.0 & 3.0 & $\begin{array}{c}0.27 \\
(\text { at } 10 \mathrm{GHz})\end{array}$ \\
\hline $\begin{array}{l}\text { Thermal conductivity: } \\
\left(\mathrm{cal} / \mathrm{cm} \cdot \mathrm{h} \cdot{ }^{\circ} \mathrm{C}\right)\end{array}$ & 0.06 & 0.06 & 0.075 \\
\hline
\end{tabular}

\section{HIGH POWER TEST}

\subsection{Alumina Disk Samples}

We used a Traveling Wave Resonator (TWR) to conduct high power tests of $\mathrm{rf}$ window alumina disks which were made with varying fractions of sintering binder $(\mathrm{MgO})$. The parameters of the seven alumina ceramic disks are summarized in Table 3.

Samples 1 to 6 had been HIP-treated. No samples had TiN coating. Sample 1 had no visible imperfections (see Fig. 10-a), but sample 2 contained impurities that looked like a dark cloud (see Fig. 10-b). Samples 3 and 5 were made of $99.9 \%$ alumina with $0.06 \% \mathrm{MgO}$ binder, and sample 4 of $99.9 \%$ alumina with no $\mathrm{MgO}$ binder. The test on sample 5 was done to study the reproducibility of the result of sample 3. Sample 6 had ten 0.5-mm-diameter holes through it. Sample 7 was made of the same material as samples 3 and 5 except for the porosity $(0.8 \%)$, which was 1.6 times as large, because samples 3 and 5 had been HIP treated. Samples 6 and 7 were made to investigate the relation between micro discharge breakdown power and porosity size at the grain boundaries of alumina ceramic.

Table 3: Parameters of alumina disks tested in this experiment.

\begin{tabular}{|l|c|c|c|c|c|}
\hline & $\begin{array}{c}\text { Purity } \\
(\%)\end{array}$ & $\begin{array}{c}\text { Prosity } \\
(\%)\end{array}$ & $\mathrm{MgO}(\%)$ & $\begin{array}{c}\text { Loss } \\
\left(10^{-4}\right)\end{array}$ & $\begin{array}{c}\text { Visible } \\
\text { impurity }\end{array}$ \\
\hline$\# 1$ & 99.5 & 0.5 & 0.19 & 12 & no \\
\hline$\# 2$ & 99.5 & 0.5 & 0.19 & 12 & yes \\
\hline$\# 3$ & 99.9 & 0.5 & 0.06 & 3 & no \\
\hline$\# 4$ & 99.9 & 0.5 & 0.00 & 0.3 & no \\
\hline$\# 5$ & 99.9 & 0.5 & 0.06 & 3 & no \\
\hline$\# 6$ & 99.9 & 0.5 & 0.06 & 3 & no \\
\hline$\# 7$ & 99.9 & $0.8^{(2)}$ & 0.06 & 3 & no \\
\hline
\end{tabular}

(1) This sample had ten $0.5 \mathrm{~mm}$ diameter holes through it. (2) No HIP treatment.

\subsection{Experimental Results}

Figure 11 summarizes the results of the experiment, showing the observed temperature rise at the window frame as a function of the average power for each sample.

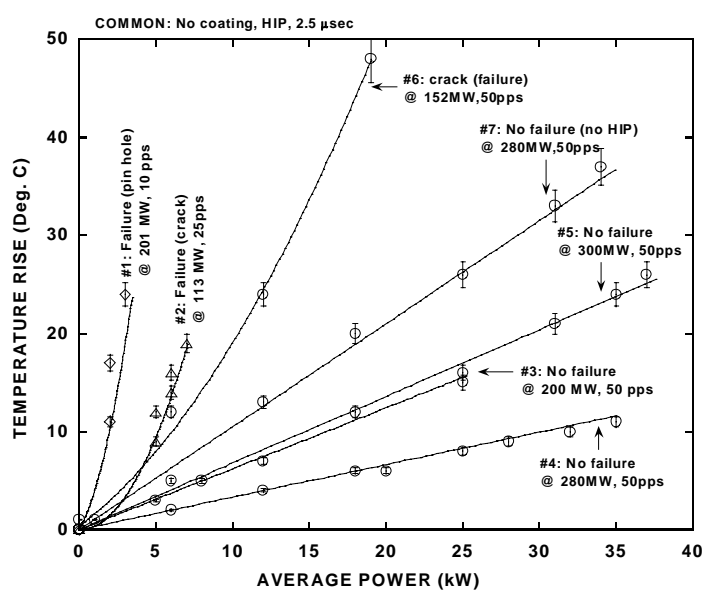

Figure 11: Experimental results. No samples had any surface coating. Samples 1 to 6 had HIP treatment. Sample 7 was made from fine grain alumina powder (see Fig. 7).

Sample 1: During the operation, localized light emission from a single spot increased continuously. At a transmission power of $200 \mathrm{MW}$, a large discharge took place and the disk was destroyed. Electron microscopy showed numerous melted pin-holes with diameters of $\sim 10$ $\mu \mathrm{m}$ at the point where the light emission was seen, along with a few-cm-long crack. At a power transmission of 200 MW, multipactoring does not cover the entire window surface. Our interpretation is that a highly localized multipactor started at a defective spot on the surface, and the resultant electron collisions on the surface became the heat source causing the disk failure.

Sample 2: No localized discharge was seen during the test. Sample 2 did not experience a steep temperature rise, as did sample 1 . At $25 \mathrm{~Hz}$, when the power reached 113 MW, the alumina disk cracked and was destroyed. No trace of melting was seen on the surface. We interpret this failure as having been caused by heating due to the localized sintering binder (dark cloud).

Sample 3: The test was continued up to $280 \mathrm{MW}$ at 25 $\mathrm{Hz}$, then up to $200 \mathrm{MW}$ at $50 \mathrm{~Hz}$, and terminated at that point. The alumina disk showed no damage. The temperature rise was small and the operation very stable.

Figure 5 shows the light emission profile from sample 3 during the test. The light signal trace is synchronized with the input rf power. At the rising and falling edges of the rf pulse, when the power transmission approaches 10 MW, light emissions for a few hundred nano-seconds are clearly seen. Note that our samples do not have a TiN coating. Although the operational power was well above the multipactoring regime, during the pulse transient time the disk momentarily experienced power levels where multipactoring is prominent. Therefore, for $\mathrm{rf}$ window disks in practical use, TiN coating is required to reduce the charge up on the alumina ceramic disk due to the electrons multipactoring during the pulse transient time.

Figure 12 shows the time profile of $\mathrm{X}$-ray emission from sample 4. The amount of X-ray emission was roughly proportional to the power transmission through 
the window. This figure shows typical behavior of sample 4, when no discharge was taking place. As stated earlier, multipactoring is pronounced only during the pulse transient time, resulting in electrons with a relatively low energy (a few $\mathrm{keV}$ ). Although they may also emit X-rays, the photon energies are too small to penetrate the window frame. Those soft X-rays are not observed by outside detectors.

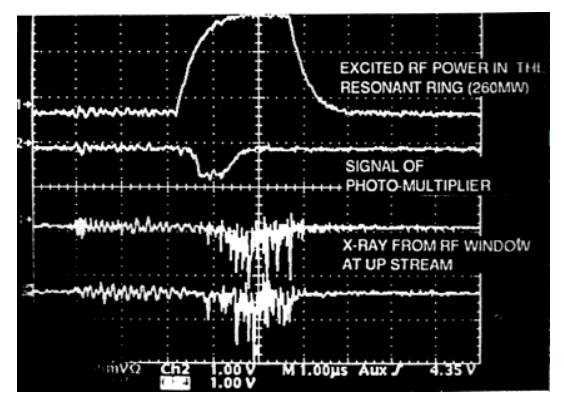

Fig. 12: Time profiles of rf power, fluorescent light, and X-ray emission from sample 4 during stable operation.

Sample 4: No failure occurred. Sample 4 showed very stable performance with the lowest temperature rise even at the maximum power of $280 \mathrm{MW}$ at $50 \mathrm{~Hz}$.

Sample 5: No failure occurred at $300 \mathrm{MW}$ at $50 \mathrm{~Hz}$. Figure 9 shows that the temperature rise in sample 5 was very similar to that in sample 3. No melting spots or cracks were found on the disk.

Sample 6: Continuous discharging occurred at some pin holes we had made in the sample during rf operation above $50 \mathrm{MW}$ transmission power. With a transmission power of $152 \mathrm{MW}$, a large discharge took place, and the disk was destroyed (cracked). Sample 6 showed the highest temperature rise among the samples tested.

Sample 7: No failure occurred at $280 \mathrm{MW}$ and $50 \mathrm{~Hz}$ The temperature rise of sample 7 was 1.5 times as high as that of samples 3 and 5 .

\subsection{Discussions}

What we have learned from these tests can be summarized as follows:

When there is a surface defect, it can initiate localized multipactoring. The surface temperature at the spot is increased. Then the secondary emission coefficient of the alumina is increased, and the thermal heat conductivity is decreased in proportion to the temperature rise. This process self-amplifies rapidly until the alumina disk is destroyed (sample 1). When there is an internal defect due to localized concentrations of sintering binders or voids, it can cause heat build-up at that spot and the thermal strain can cause cracking (sample 2). Clearly temperature rise depends on the degree of porosity of the alumina ceramic disk (sample 7). The high power performance of alumina disks can be dramatically improved by using HIP on highpurity alumina (samples 3, 4, and 5). By not adding $\mathrm{MgO}$ as the sintering binder for alumina, the dielectric rf loss is decreased and the rf heating is decreased. This also contributes to the stability of alumina disks in high power operation (sample 4).

\section{CONCLUSIONS}

A computer simulation of multipactoring electrons on the rf window disk surface was conducted for a theoretical study of the breakdown mechanism. Experiments using a TWR were carried out to study the high power behavior of window disks made of high purity alumina with HIP treatment, but without TiN coating. Over a wide range of transmission power, good agreement was found between the calculated distribution of multipactoring electrons hitting the alumina surface and the experimentally observed fluorescence pattern on the window. The simulation predicts that multipactoring will not take place at transmission powers above $50 \mathrm{MW}$. Observations in the TWR experiment confirmed this prediction. Even at this power level, however, multipactoring was found to occur during the rf pulse transient time. Therefore, coating with TiN is necessary to maintain the best possible overall characteristics of alumina disks for $\mathrm{rf}$ windows. At a power level above the multipactoring region, imperfections inside the alumina ceramic disk are the dominant source of breakdowns. As a cure, we have developed a technique based on the following combination:

1) use of high-purity ultra fine alumina powder, 2) avoidance of $\mathrm{MgO}$ sintering binder, and 3) HIP treatment.

This significantly improves the mechanical properties of alumina, and reduces the dielectric RF losses and the porosity. The high power performance of alumina disks fabricated this way is significantly superior to that of disks made in the traditional way. The optimization of alumina ceramic disk fabrication and testing for long-term performance of a large number of disks requires future work. Such studies will be continued in order to build a basis for the design of rf windows for future accelerators.

\section{REFERENCES}

[1] D. H. Preinst, IRE Trans. ED-8, 243 (July 1961).

[2] R. Hayes, Final Report, AD 256259, Eitel-McCullough Inc., San Carlos, June 1964.

[3] R. Nyaiesh, E. L. Garwin, F. K. King and R. E. Kirby, Proc. 32nd National Symp. of American Vacuum Society, Houston, November 1985.

[4] R. B. Neal, D. W. Dupen, H. A. Hogg and G. A. Loew, "The Stanford Two mail Accelerator", W. A. Benjamin Inc., 1968.

[5] Frank Kriensen, SLAC/AP-23, 1984.

[6] Y. Saito, S. Yamaguchi, S. Anami, S. Michizono, A. Kinbara and N. Matsuda, Proc. 14th Int. Sym. on Discharge and Electrical Insulation in Vacuum, September 1990.

[7] Y. Saito, N. Matsuda, S. Anami, A. Kinbara, G. Horikoshi and J. Tanaka, IEEE Trans. Electrical Insulation, 24 (6), p. 1029-1032, (1989).

[8] S. Michizono Y. Saito, S. Anami, A. Kinbara, et al., Proc. 16th Int. Symp. on Discharge and Electrical Insulation in Vacuum, 1992.

[9] A. Miura and H. Matsumoto, Proc. of Int. Conf. for High Energy Accelerators, 1992.

[10] S. Yamaguchi, Y. Saito, S. Anami and S. Michizono, IEEE Trans. Nucl. Sci. NS-39, (1992).

[11] A. Miura and H. Matsumoto, Proc. Int. Linear Accelerator Conf. Ottawa, 1992.

[12] A. Miura and H. Matsumoto, NIM A334 (1993) 341-352.

[13] W. R. Fowkes, R. S. Callin and M. Studzinski, Proc. IEEE Part. Accel. Conf., San Fransico, 1991. 